\title{
Die Gefahren und der Nutzen der intrauterinen Injectionen.
}

Von

\section{P. Zweifel in Leipzig.}

(Mit 2 Textfiguresi.)

Man muss sich wundern, dass in der Medicin manchmal Gegensätze da auftreten, wo es sehr leicht erscheint, durch die Thatsachen das Richtige zu beweisen: wie z. B. bei diesen Injectionen. Es handelt sich darum, ob Arzneilösungen bei intrauterinen Einspritzungen durch die Tuben in die Bauchhöhle fliessen können und in einer gewissen Regelmässigkeit überfliessen oder nicht.

Ganz àbgesehen von einzelnen Beobachtungen, die im Sinne der Bejahung schon früher gedeutet wurden, liegen unmittelbar beim lebenden Menschen ausgeführte Versuche vor, welche einander schnurstracks widersprechen.

Von der Mehrzahl der Autoren wird die Lehre in wenig sagender Halbheit wiedergegeben, das Ueberfliessen meist im Princip verneint, aber für die Praxis doch diejenige Vorsicht empfohlen, welche für den Fall der Möglichkeit nöthig wäre, um ein Unglück zu verhüten. So ungefähr lautet die oft zu lesende Mittelwegsfassung.

Mit bedingungsloser Zuversicht vertrat Döderlein ${ }^{1}$ ) auf dem 7. Congress der deutschen Gesellschaft für Gynäkologie in Leipzig den Satz, dass bei solchen Einspritzungen eine grosse Menge Flüssigkeit in die Bauchhöhle überlaufen könne, denn er beob. achtete eine Frau, die schwanger zu sein glaubte, aber es nicht war und die zum Zweck der Abtreibung eine der dünnen, langen, berüchtigten Spritzenröhren unter Führung ihres Fingers in den

1) Verhandlungen der deutschen Gesellschaft für Gynäkologie. Congress in Leipzig 1897. S. 432. 
Cervicalcanal einführte und eine Spülkanne voll Lysol einfliessen liess. Vom Lysol floss wenig zurück, und der Frau wurde es so übel, dass sie zu sterben fürchtete. In ihrer Todesangst schickte sie nach einem Arzt und Döderlein, der die Kranke ebenfalls zu sehen bekam, konnte in der Bauchhöhle eine grössere Menge frei beweglicher Flüssigkeit durch Percussion nachweisen. Die Frau überstand die Katastrophe.

An diesen einen Fall schloss Döderlein Versuche mit Farblösungen bei Uteris an, welche wegen irgend welcher Erkrankungen exstirpirt werden sollten und fand in 1 von 4 Fällen die Farblösung in einer Lache im Douglas, bei zweien waren die Tuben verschlossen, bei der Vierten war nichts übergetreten. Von der Jodtinctur floss nichts in die Bauchhöhle, von einer 50 proc. Chlorzinklösung war sichtlich ein Theil übergegangen.

Auch ich unternahm solche Spülungen mit Farblösung bei jeder abdominellen Uterusexstirpation wegen Portiocarcinom, die gleich 2 Zwecke zu erfüllen hatten: erstens die Gebärmutterhöhle zu desinficiren und die Frage des Uebertrittes der Flüssigkeit in die Tuben und die Bauchhöhle zu entscheiden.

Meine Versuche waren im Sinne des Uebertrittes positiv ausfallen und wurden in einem diesbezüglichen Aufsatz der deutsehen medicinischen Wochenschrift ${ }^{1}$ ) erwähnt, stiessen jedoch bei Tho rn $^{2}$ ) auf entschiedenen Widerspruch, weil er bei gleichen Versuchen völlig entgegengesetzte Ergebnisse erbalten hatte. Er spritzte 5 Mal Jodtinctur mit der Braun'schen Spritze in die Gebärmutter ein ohne ein Ueberfliessen zu sehen und nur bei einem 6. Versuch fand er bei Verwendung einer wässerigen Methylviolettlösung eine Färbung des Interstitium der linken Tube.

Die Veröffentlichung 'Thorn's hat diese Lehre auf einen todten Punkt gebracht, sodass kein Mensch mehr weiss, was richtig ist. Und doch bedarf sie dringend der Aufklärung, weil bei der Möglichkeit des Ueberfliessens Vergiftungen und Verätzungen mit ihren schweren Folgen zu Stande kommen können, während die intrauterinen Spülungen bei der Unmöglichkeit, die Thorn behauptet, keine Gefahr bedingen. Es ist der Entscheid sehr wichtig, da die Aerzte von Thorn direct aufgefordert wurden, unbesorgt vor Gefahren die Intrauterininjectionen anzuwenden.

1) Deutsche med. Wochenschr. 1904. No. 17.

2) Centralbl. f. Gyn. 1904. No. 38. S. 1128. 
$300 \mathrm{Zweifel}$, Die Gefahren und der Nutzen der intrauterinen Injectionen.

An mich war der Widerspruch gerichtet, obschon ich nur der letzte der Autoren war, der das Ueberfliessen vertrat; aber die Ungewissheit erregte bei mir den festen Willen die Frage durch eine methodische Prüfung zu klären, doch ausschliesslich durch neue Thatsachen, was um so mehr angezeigt war, als sich hier Experimente in viva mit einer durchaus zweckmässigen Behandlung verbinden lassen.

Wir pflegen schon seit langer Zeit bei allen Exstirpationen des carcinomatösen Uterus eine desinficirende Injection in dessen Höhle vorauszuschicken, nicht etwa, weil wir in die alte Lehre zuräckgefallen wären, dass die Gebärmutterschleimhaut gesunder Frauen keimhaltig sei, sondern allein zu dem Zweck, niemals eine Verhaltung von Eiter oder Jauche, wie das bei Cervixcarcinomen vorkommt, unentdeckt $z u$ Jassen. Das Uebersehen einer solchen Pyometra hat selbstverständlich das Platzen und den Eiteraustritt zur ungelegenen Zeit, nämlich bei offener Bauchhöhle zu Folge und fübrte in einem unserer eigenen Fälle zur tödtlichen Peritonitis.

Dem Einwand, dass wir solche therapeutische Spülungen nicht zu Schlüssen über das normale Organ verwerthen dürfen, begegnen wir mit dem Hinweis, dass gerade bei Portiocarcinomen der Fundus uteri und die Adnexe in der Regel anatomisch völlig gesund sind, sodass es in unserer Macht liegt, die zur Beweisführung geeigneten Fälle auszusuchen. Am besten passen dazu die Portiocarcinome, weniger die Cervixearcinome, weil diese durch die Gewebszerstörung oft eine weite Höhle bilden, in denen sich das Spülrohr verfängt und die Flüssigkeit unter die Serosa leitet. Es war uns in einigen Fällen von Cervixcarcinom unbeabsichtigt eine Färbung der Lymphwege und der nächsten Lymphdrüsen gelungen.

Solche Spülungen mit Pyoktaninlösung (Methylviolett) von 1 pM. und dem Fritseh-Bozemann'schen Katheter nahmen wir nach der Thorn'schen Veröffentlichung wieder auf.

Gleich bei den ersten Versuchen im Herbst 1904 fanden wir bei der Eröffnung der Bauchböhle 2 Mal die Farblösung in grossen Lachen im Douglas'schen Raum, sodass die Tupfer tiefblau zurückkamen und selbstverständlich die beiden Tuben in ihrer ganzen Länge gefärbt waren, also eine volle Bestätigung von Döderleins's und unseren eigenen Versuche ergaben. Ein drittes Mal war die Farbe sogar in 2 am Fransenende verlötete, weite 
Eileiter eingeflossen und entleerte sich beim Lostrennen der Verwachsungen im Schwall.

Aber bei weiteren Versuchen nahm die Regelmässigkeit des Durchfliessens und die Menge der durehgeflossenen Farblösung ab, obschon wir dieselbe Concentration beibehielten. Die eine oder die andere Tube war wohl noch gelegentlich auf dem Durchschnitt blau, aber durch das ganze Wintersemester 1904/05 hatte sich niemals wieder eine Ansammlung im Douglas'schen Raum gebildet.

Man pflegt bei so widerspruchsvollem Ergebniss gern des Zufalls launenhaftes Spiel anzunehmen und stand auch ich in Gefahr, in die banausische Mittelwegsfassung einzustimmen: es kann so sein und meistens ist es anders. An des Zufalls Spiel konnte ich jedoch in diesem Fall nicht glauben und weil ein gewisser Reiz darin lag, herauszubringen, unter welchen Verhältnissen ein Ueberfliessen einer wässeriger Farblösung zu Stande komme und wann nicht, weil mit der Kenntniss der Bedingungen auch ein Mittel an die Hand gegeben war, um solche Uebergänge zu verhüten oder zu begünstigen, beschloss ich, weiter zu prüfen, und da die Erprobung Zeit hatte, wurde Jahre lang in jedem passenden Falle von Carcinoma uteri die Pyoktanineinspülung mit dem FritschBozemann'schen Katheter fortgesetzt.

Zuerst wurde darauf geachtet, dass die Spülflüssigkeit körperwarm und indifferent war, also Methylviolett in körperwarmer 0,7 proc. Kochsalzlösung. Das änderte nicht viel. Später wurde streng darauf geachtet, dass der Uterus vor der Spülung nicht mechanisch gereizt und dadurch zu Contractionen gebracht war; denn es fiel mir auf, dass die Färbung ausblieb, wo die intrauterine Spülung erst nach der Auslöffelung und Verschorfung des Careinoms als letzter Act dor Desinfection vorgenommen wurde.

Vom Mai 1905 ab wurde diese Aenderung eingeführt und die Spülung vor der Excochleation und Combustion vorgenommen, was das Ergebniss im Laufe eines weiteren Jahres so änderte, dass auf 36 Fälle 13 Mal die Flüssigkeit durch die Tuben floss und 23 Mal nicht.

Wie die Zahlen sagen, blieb das Ueberfliessen in der Mehrzahl der Fälle aus und doch war es viel häufiger geworden als im Jahre vorher. Es galt darum noch mehr darauf bedacht zu 
sein, alle Reizungen mechanischer, thermischer oder chemischer Art auszuschalten.

Vom Mai 1906 an wurde befohlen, die Frau vorher nieht bimanuell zu untersuchen, ja in der letzten Zeit wurde auch die Scheidendesinfection abgestellt und erst nach der Pyoktanimeinspülung vorgenommen.

Auf weitere 33 Fälle von Ostern 1906-1907 war das Ueberfliessen so regelmässig, dass nur noch 8 negative Fälle auf 25 positive kamen. Aber es genügte die versehentliche Ausführung der Scheidendesinfection von Seiten einer neuen Schwester, dass in diesen Fällen kein Tropfen der Hethylviolettlösung in die Tuben überging.

Von Ueberfliessen wurde gesprochen, wenn auch nur eine Tabe bis zum Ostium abdominale blau gefärbt war, weil wir einmal bei einseitiger Färbung im Douglas'schen Raum einen EssIöffel voll verdünnter Pyoktaninlösung fanden.

Es ist dadurch sicher nachgewiesen, dass es in erster Linie die mechanischen Reizungen der Gebärmutter sind, welche das Ueberfliessen hemmen und dass nurda, wo die Gebärmutter schlaff ist und von dem Eingriff gleichsam überrascht wird, die Farbe in der Regel durchfliesst.

Ein paar Fälle fielen negativ aus, weil die Eileiter besonders eng oder ganz verschlossen waren, und haben wir auch Fälle zu verzeichnen, bei denen die Flüssigkeit durch den einen weiten Eileiter bis in die Bauchhöhle floss, der andere aber ganz ungefärbt blieb, weil er eng war. Das Entscheidende war in der Regel: schlaff oder straff, während alle anderen Verhältnisse weniger Unterschied machten, so auch das Alter der Frauen.

Es kann nach dem Ergebniss unserer zahlreichen intraterinen Spülungen, mit Untersuchung der ausgeschnittenen Tuben, nicht länger bestritten werden, dass wässerige Lösungen, die nicht ätzen, nicht reizen und unter der Bedingung, dass jede Anregung zu Zusammenziehungen der Gebärmutter vermieden ist, in der Regel in die Bauchhöhle überfliessen.

Vom Mai 1907 an wurde Jodtinctur ausgeprüft, um zu sehen, ob nicht der Unterschied zwischen meinen Ergebnissen und denen von Thorn von dem starken chemischen Reiz abhängig sei, den die Jodtinctur ausübt. 
Als wir diese Einspritzungen begannen, überraschte uns gleich der erste Fall, weil die Jodtinctur in beide Eileiter eingeflossen war. Wir setzten diese Einspritzungen mittelst einer Pravatz'schen Spritze, an welche ein besonders dünnes, schmales Kanülenrohr (siehe Figur 1) angeschraubt wurde, noch einige Male fort, erhielten noch $3 \mathrm{Mal}$ einige Centimeter weit eine Färbung mit Jod, aber manchmal wegen der Unbestimmtheit der gelben Färbung ein so ungewisses krgebniss, dass wir uns entschlossen diese Spülungen abzuändern. Die Jodtinctur ist zu beweisenden. Versuchen nicht geeignet, erstens weil hier zwei Reize zusammenwirken, das Jod und der Alkohol, zweitens weil die Jodtinctur auf der weichen Uterusschleimhaut ausgedehnte Krümel bildet, welche die Tinctur absorbiren und ein Ueberfliessen in die Tuben erschweren und drittens wegen der wenig charakteristischen Färbung der Gewebe.

Um zu beweisenden Ergebnissen zu gelangen über die Frage, ob der Alkohol einen so starken chemischen Reiz ausübe, dass durch ihn die Verschiedenheit der Ergebnisse zu erklären sei, kam ich wieder zu dem Methylviolett und dem BozemannFritsch'schen Katheter zurück, nur mit dem Unterschied, dass statt der $0,7 \mathrm{pCt}$. Kochsalzlösung $200 \mathrm{~cm}$ Alkohol von $96 \mathrm{pCt}$. genommen wurden.

Die Einspritzungen mit dieser Anordnung brachten Fall für Fall Färbungen der Tuben zu Stande und lieferten den Beweis, dass auch alkoholische Lösungen bei genügender Flüssigkeitsmenge und bei Unterlassung jeder mechanischen Reizung regelmässig in die Eileiter überfliessen. Unter 11 Versuchen waren nur 2 negativ. Selbst bei den verschlossenen Tuben war die alkoholische Methylviolettlösung bis zur Verwachsungsstelle vorgedrungen.

Doch war ein Unterschied gegenüber den wässerigen Lösungen nicht zu verkennen, indem der den Katheter führende Finger kurze Zeit nach dem Einfliessen eine leichte Umschnürung des Instrumentes durch den sich zusammenziehenden Uterus verspürte und bei keinem der vielen Versuche je ein Ueberfliessen bis in den Douglas'schen Raum zu Stande kam. Der ehemische Reiz macht sich bemerkbar, aber er ist nicht so stark und wirkt nicht so rasch, dass er den Uebertritt von Flüssigkeit in die Eileiter zu verhindern vermag. 
$304 \mathrm{Zweifel,} \mathrm{Die} \mathrm{Geíahren} \mathrm{und} \mathrm{der} \mathrm{Nutzen} \mathrm{der} \mathrm{intrauterinen} \mathrm{Injectionen.}$

Nachdem durch die vielen Versuche mit wässerigen und alkoholisehen Lösungen der Uebertritt von Flüssigkeit bewiesen war, liess sich gewiss ungezwungen daraus schliessen, dass auch der Liquor ferri sesquichlorati, dem man eine ganze Reihe von traurigen Unglücksfällen zur Last legt, durch die Tuben fliessen könne. Weil jedoch in der Medicin die Verallgemeinerung von Schlüssen schon oft auf den Holzweg geführt hat und da der Unterschied zwischen einer wässerigen Methylviolettlösung und der riesig sauern, ätzenden Chloreisenlösung ein mehrfacher ist, nahm ich mir vor, die Verallgemeinerung des Schlusses nicht zu wagen, ohne dieses Medikament auch versucht zu haben.

Der Versuch gab der Erwartung recht, denn es floss der Liquor ferri sesquichlorati ausgiebig in die Tuben ein, selbst da, wo die Flüssigkeitsmenge noch nicht einmal $1 \mathrm{ccm}$ betrug und die Gebärmutterhöhle recht geräumig war.

Ich bin mir immer bewusst, dass ich mit diesen Ergebnissen in Widerspruch mit einer ausgedehnten Erfahrung komme, da doch die Intrauterininjectionen hundert- und tausendfältig von Frauenärzten ausgeführt wurden. Aber es wird sich die Praxis, bei welcher keineswegs mit gleicher Consequenz verlahren wird, nach den Ergebnissen dieser methodischen Versuche zu richten haben, nicht umgekehrt, um so mehr als die Methode dieser Versuche recht gut erklärt, warum die an sich gefährlichen Injectionen mit dem ätzenden Liquor ferri sesquichlorati doch nur in seltenen Ausnahmefällen ein Unglück angerichtet haben.

Der Gedanke, dass die Intrauterinspülungen gefährlich werden können, ist nicht neu und haben schon viele Autoren sich damit beschäftigt, ihnen die Gefahr zu benehmen.

Carl v. Braun gab zum Schutz gegen das Ueberfliessen in die Tuben grundsätzlich den Rath nur 2-4 Tropfen zu verwenden, d. i. $0,1-0,2 \mathrm{ccm}$. Andere Autoren schlugen vor, die Einspritzungen nur mit sogenannten Pinselspritzen auszuführen, d. h. mit Intrauterincanülen, welche von Watte umwickelt waren, damit ein Ueberschuss des Medicamentes aufgefangen und vor dem Eintreten in die Tuben bewahrt werde. Chrobak ${ }^{1}$ gab in seiner Bearbeitung der allgemeinen Gynäkologie einen solchen Spritzenansatz von $\mathrm{H}_{0} \mathrm{f}$ -

1) Chrobak im Handbuch der Frauenkrankheiten von Billroth und Lǘcke. 2. Aufl. 1885. Bd. I. S. 176. 
$\operatorname{mann}^{1}$ ) mit Abbildung an. Wir construirten zu weiteren Versuchen eine Spritzencanüle (s. Fig. 1), die wir in der Breite der bekannten amerikanischen Silberstäbchen und möglichst platt hatten anfertigen lassen und umwickelten dieselbe mit hydrophiler Watte. Trotz dieser Vorsicht floss der Liquor ferri, den ich in der Menge von $1 \mathrm{ccm}$ in die Uterushöhle einspritzte und trotzdem sich die Watte bis unter den äusseren Muttermund hinunter mit Liquor vollsaugte, also denselben theilweise ableitete, in beide Tuben ein und durch die offene linke bis in die Bauchhöhle, in der rechten bis zu einer Verwachsung, welche unmittelbar vor dem Ostium abdominale tubae war. Also trotz dieser Vorsicht und trotz der geringen Menge von Flüssigkeit kam ein Ueberfliessen vor, welches entsprechenden Falles bei der intrauterinen Behandlung eine lebensgefährliche oder :ödtliche Verätzung erzeugt hätte.

Figur 1.

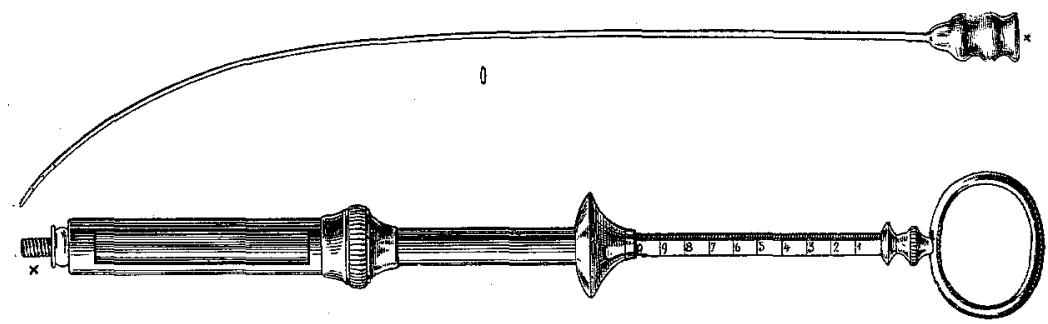

Intrauterinspritze mit flacher Kanüle und 6 feinen seitlichen Oeffnungen.

Ungefähr halbe natürliche Grösse.

Es geht ans diesen Versuchen mit zwingender Nothwendigkeit der Schluss hervor, dass man in das Corpus uteri überhaupt keine ätzende Flüssigkeit einspritzen darf, oder nur 2-4 Tropfen, wenn der Abfluss nicht vollkommen gesichert ist.

Folgerichtig läuft diese strenge Einschränkung der intrauterinen Einspritzungen auf eine Empfehlung der Pinselbehandlung hinaus.

Auch diese lässt sich selbstverständlich mit dem gleichen Verfahren prüfen. Zuerst benutzte ich dazu wieder Methylviolettlösung, später aber, weil die Arzneiwirkung besser erkennbar ist, den Liquor ferri sesquichlorati, indem ich immer zwei Menge'sche Stäbchen nahm, sie in die Farbflüssigkeit tauchte und möglichst ausgiebig nach den beiden Hörnern des Uterus schob, selbstverständlich unter

1) Hoffmann, Wiener med. Presse. 1878. No. 9 ff. 
den gleichen Bedingungen, dass der Uterus vor jeder mechanischen Reizung verschont war.

Die Besichtigung der Gebärmutterhöhle nach der Exstirpation zeigte auf 24 Fälle 8 mal ein positives Ergebniss, 10 mal war keine Spur von Liquor im Corpus uteri, 6 mal nur ein schmaler Streifen in einer Furche der Schleimhaut, die das Stäbchen gegraben hatte, gefärbt, die übrige Fläche unbenetzt. Es war bald klar, dass die Färbung und Verbreitung des Liquor in der Höhle des Corpus dann gelingt, wenn der Muttermund weit ist, dagegen alles ausgepresst wird, man mag die Pinsel drehen und wenden wie man will, wo der innere Muttermund eng ist. Natürlich wäre in den letzteren Fällen eine Arzneiwirkung auf das Corpus uteri durch Pinseleinführung illusorisch gewesen.

Es geben diese Versuche eine theilweise Bestätigung für die Ansicht früherer Autoren, so insbesondere Hofmeier"s, in einer mir selbst überraschenden Weise und erklären manche Erfahrungen der gynäkologischen Praxis, so z. B. die sehr guten Erfolge der Pinselbehandlung gegen die Cervixkatarrhe mit der starken Schleimabsonderung and die fast völlige Eriolglosigkeit der Bepinselung. mit Liquor ferri gegen die Blutungen.

Nach diesen Untersuchungen ist die für wässerige Lösungen ausgesprochene Durchgängigkeit der Eileiter auch füralkoholische und für ätzende wässerige Lösungen vorhanden, andererseits ist bei der Pinselbehandlung nur ausnahmsweise, d. h. nur bei weitem Halseanal, darauf zu rechnen, dass die angewandten Arzneien in das Corpus uteri gelangen und da die ätzenden Lösungen, wenn sie in die Bauchhöhle vordringen, heftigste Schmerzanfälle, ja sogar tödtliche Peritonitis bedingen können, ist dringend davor zu warnen.

Rückwärts können wir über die tausendfältig ohne schlimme Folgen gemachten Spülungen und Einspritzungen schliessen, dass in den gut verlaufenen Fällen unbewusst die Gebärmutter mechanisch gereizt und sie, wie die Eileiter fest zusammengezogen waren. Wenn auch ein kräftiges Massiren des Uterus ein fast sicheres Mittel ist, um das Ueberfliessen zu. verhindern, so bleibt es doch gewagt, ätzende Flüssigkeiten in grösserer Nenge als 3-4 Tropfen in die Gebärmutterhöhle einzuspritzen, ja selbst bei so wenig sahen wir einmal eine Peritonitis entstehen, und werden es nicht mehr thun.

In der Literatur sind Peritonitiden, die nach Injectionen mit. 
Liquor ferri sesquichlorati entstanden, in grösserer Zahl beschrieben und führt (hrobak ${ }^{1}$ ) in seinem ausgezeichneten Werke, das oben citirt ist, 14 tödtlich abgelaufene Fälle an, von denen diejenigen von Haselberg, Gubiau, Kern, Kormann und je 2 von Bequerel und von Hegar-Kaltenbach als beweisend für den Uebertritt des Liquor ferri in die Bauchhöhle anerkannt wurden. Menge $\left.{ }^{2}\right)$ spricht von 25, aber sicher entspricht die Zahl der publicirten Fälle keineswegs der Wirklichkeit, sondern es sind hunderte vergekommen, aber nicht veröffentlicht, sondern in stillem Gram verschwiegen worden. Das kann ich aus eigener Erfahrung ermessen, da ich von 5 Fällen weiss, die ich in Consultation oder durch Erzählung anderer Aerzte in den Einzelheiten erfahren habe, von denen keiner veröffentlicht ist.

Bemerkenswerther Weise widersetzt sich Chrobak dem Schluss, dass man die Einspritzungen mit Eisenchlorid einstellen solle und erhebt gegen alle $\mathrm{jhm}$ bekannt gewordenen Todesfälle den Vorwurf, dass sic mit ungenügenden Instrumenten und unter Ausserachtlassung der nöthigen Vorsicht ausgeführt worden seien.

Man muss fragen, warum von einem so ausgezeichneten Frauenarzt wie Chrobak an Injectionen festgehalten wurde, die solches Unglück verschuldet haben? Die Antwort lautet, weil man mit den Einspritzungen von Liquor ferri sesquichlorati ausgezeichnete Erfolge gegen Blutungen erzielen kann.

Ich selbst habe bis zu diesen Versuchen solche Injectionen in unzähligen Fällen, immer genau nach der Vorschrift von C. v. Braun ausgeführt und zwar in der Regel erlebt, dass die Kranken danach einen heftigen Schmerzanfall, manchmal auch Erbrechen bekamen, welcher Sturm jedoch immer bald vorüber ging, dass sie aber dafür belohnt wurden, indem sie Wochen oder Monate später mit freudestrahlendem Gesicht wieder kamen, um zu melden, dass die Einspritzung ausgezeichnet genützt habe, von da an die Periode ganz gering geworden sei und die starken und lange sich hinziehenden Blutverluste aufgehört hätten.

Ich leugne nicht, dass solche Erfahrungen mich immer wieder zur Anwendung eines Mittels hinzogen, welches sich nützlicher er-

1) Chrobak, Allgemeine Gynäkologie. Handbuch der Frauenkrankheiten von Billroth n. Iücke. 2. Aufl. 1885. Bd. I. S. 172.

2) Menge, Münchener med. Wochenschr. 1907. S. 1700. 
wies als die umständlicheren Ausschabungen, aber dass ich nach dem Stand der Veröffentlichungen mehrmals diese Vorliebe zurückdrängte und Jahre lang von solchen Injectionen mit Liquor ferri sesquichlorati absah. Zu dem Hin- und Herschwanken brachten. mich ausser den Veröffentlichungen anderer Autoren ganz besonders eigene Erfahrungen. Nachdem ich Jahre lang nie mehr Liquor ferri sesqu. in den Uterus, weder nach Dilatation noch nach der Abrasio, gespritzt hatte, hielt hier in der geburtshilflichen Gesellschaft Werth' ${ }^{1}$ ) einen Vortrag über die Regeneration der Uterusschleimhaut nach Ausschabung, dessen Erörterung den Anlass bot, über die intrauterinen Eisenehlorid-Einspritzungen nach den Abrasiones mucosae sich auszusprechen. Wenn ich auch Bedenken geäussert hatte, veranlassten mich die rückhaltlosen Empfehlungen von Werth und A. Martin doch von Neuem das Eisenchlorid bei Ausschabungen des Uterus zu versuchen. Aber ich erlebte danach einen Unglücksfall bei einer Frau mit kleinem. Hyoma uteri, die 3 Tage nach der Abrasio und Eisenchloridätzung fieber- und schmerzfrei im Bett lag, am 4. aufstand, am 5. heim reiste, aber schon auf dem Wege Schmerzen und zu Hause eine acute Peritonitis bekam, der sie nach kurzer Zeit erlag.

Der letzte Fall von Liquor ferri sesquichlorati-Einspritzung meiner Sprechstundenpraxis bekam trotz genauer Einhaltung von Braun's Vorschrift sofort danach einen geringen Schmerzanfall. Acht Tage später traten heftige Leibschmerzen und Fieber auf. Die Peritonitis heilte und die Kranke hatte bezüglich der Blutungen wieder einen vollen, sie ganz befriedigenden Erfolg, der mir aber doch zu theuer erkauft erschien.

Nachdem wir das Ueberfliessen nunmehr genau wissen, lässt es sich feststellen, worin die richtigen Instrumente und die richtigen Maassregeln bestehen sollen.

Zunächst sei bemerkt, dass der Rücklaufkatheter ron FritschBozemann das Ueberfliessen nicht verhindert. Zweitens, dass selbst eine vorausgehende Erweiterung des Gebärmutterhalses keine unbedingte Sicherheit verleiht gegen das Ueberfliessen und immer noch eine Gefahr in sich schliesst, wenn die Flüssigkeit stark reizend ist und wenn sie eine Contraction und Abschliessung des inneren Muttermundes bewirkt, ehe die Flüssigkeit sich hat entleeren können.

1) Sitzung rom 15. Decbr. 1894; publ. Centralbl. f. Gyn. 1895. S. 193. 
Drittens machen selbst solche Einspritzungen mit $0,1-0,2 \mathrm{ccm}$ also 2-4 Tropfen Eisenchlorid, bei denen heftige Krämpfe, ja einmal sogar eine Peritonitis entstand, den Eindruck, dass selbst so wenig Flüssigkeit in einen Eileiter einzudringen vermag, weil, wenn das Aetzen des Uterus und die Berührung des Liquor ferri sicher auf die Corpushöhle beschränkt bleibt, niemals weder ein Schmerzanfall, noch gar eine Peritonitis eintritt.

Zu diesem Schlusse von der Ungefährlichkeit einer streng auf die Gebärmutterhöhle beschränkten Aetzung führten mich folgende Erfahrungen.

Nach der Darstellung in meinen "Vorlesungen über klinische Gynäkologie" ${ }^{1}$ ) benutzte ich zum Einführen von Jodoform in den ausgeschabten Uterus starre Metallröhren. Im Anschluss an den

Figur 2.

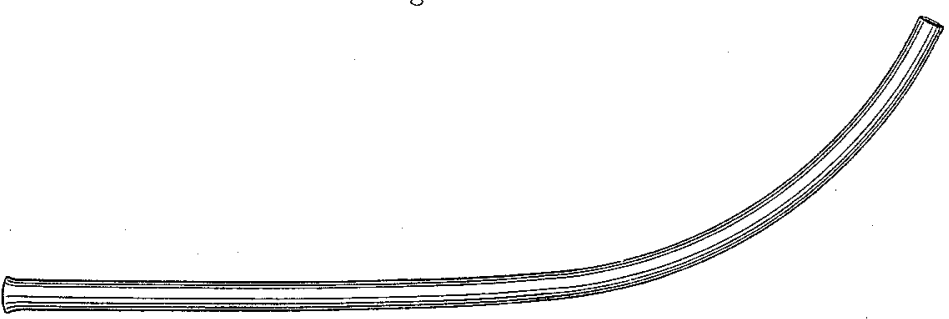

Intranterin-Röhre mit Wattepinsel. Halbe natürliche Grösse.

erwähnten Unglücksfall, der auf eine Injection mit Liquor ferri sesqu. erfolgt war, benutzte ich diese Röhren von da an immer zum Eingiessen des Liquor ferri sesquichlorati. Ein Wattepinsel auf dreifach gedrehtem Eisendraht, der durch das Rohr nachgeschoben wird, hat den Zweck, die Flüssigkeit aufzusaugen und mit allen Theilen der Gebärmutterhöhle in Berührung zu bringen, um schliesslich beim allmählichen Zurückziehen des starren Robres das Chloreisen sicher nach unten abzuleiten.

In vielen hunderten von Fällen ist bei dieser Anwendung des Eisenchlorids niemals die geringste Störung, nie ein Krampfanfall, niemals eine Entzündung

1) 1892 . S. 414 u. 415 . 
$310 Z$ Weifel, Die Gefahren und der Nutzen der intrauterinen Injectionen.

des Bauchfells vorgekommen. Diese ist nichts anderes als cine Pinselbehandlung mit Sicherheitsvorrichtungen, bei welcher durch das eingeführte starre Metallrohr einerseits verbürgt wird, dass die Flüssigkeit in den Fundus uteri gelangt, andererseits, dass sie nach dem Benetzen und Verbreiten durch den Wattepinsel, wieder zurückfliesst und dass nie ein höherer Druck auf die Flüssigkeitssäule wirken kann als der ihrer Höhe. Auch Uteruscontractionen können die Flüssigkeit nicht in die Tuben treiben, denn ehe sich der innere Muttermund zusammenziehen kann, ist jeder Ueberschuss entfernt.

Es macht einen eigenthümlichen Eindruck, dass bei dieser vielhundertfachen Anwendung des Liquor ferri sesqu. niemals etwas Schlimmes auftrat, nie ein Schmerzanfall und bei viel weniger Fällen von vorsichtiger Anwendung der Braun'schen Spritze regelmässig Schmerzen und einmal eine Peritonitis. Daraus ziehen wir den Schluss, dass ohne den unbedingt freien Abfluss des Ueberschusses viel häufiger, als wir bisher angenommen haben, ein Uebertritt der eingespritzten Medikamente in die Tuben stattfand.

Nun hat aber diese Pinselbehandlung mit gesichert freiem Rückfluss des Ueberschusses die Schattenseite, dass der Gebärmutterhals mit Quellstift oder Dilatatorium irgend einer Art vorher erweitert werden muss und sich dadurch die Einspritzung viel umständlicher gestaltet, sodass sie in der Regel nicht mehr in der Sprechstunde auszuführen ist.

Es ist oben erwähnt, dass in einem Drittel der untersuchten Frauen, die allerdings immer Mehrgebärende waren, die Wattepinsel so leicht durch den inneren Muttermund gingen, dass derselbe die Flüssigkeit nicht auspresste. In solchen Fällen lassen sich auch kurze starre Röhren bis in den Fundus uteri einführen und durch dieselben bindurch die Pinselätzung bezw. die Intrauterininjection vollziehen. Aber auch ohne diesen Sicherheitsabfluss wird es bei so weitem Gebärmutterhals durch die einfache Pinselbehandlung keinen Uebertritt der Flüssigkeit in die Tuben geben.

Es ist also nur da, wo der innere Muttermund eng ist, eine Erweiterung des Cervicalcanals, sei es durch Schnelldilatation oder durch Quellstifte nöthig und nur da die umständlichere Behandlung angezeigt. Man hat diese Einspritzung in den $70 \mathrm{er}$ und $80 \mathrm{er}$ Jahren des letzten Jahrhunderts immer mit Dilatation gemacht. Man 
wird es auch jetzt bei den Frauen mit engem Muttermund grundsätzlich wieder thun müssen, um ihnen die Gefahr einer Bauchfellentzündung zu ersparen.

Diese erhöhten Vorsichtsmaassregeln sind nur nöthig für ätzende Arzneilösungen, obenan den Liquor ferri sesquichlorati, Chlorzink und dergl., deren Ueberfliessen in die Eileiter die stürmischen Koliken und sogar echte eitrige Entzündungen verschulden können.

Wir können an der Frage nicht vorübergehen, wie es denkbar ist, dass eine aseptische keimfreie Flüssigkeit, wie der Liquor ferri sesquichlorati, selbst wenn man ihn noch vorher kocht, im Stande sei eine Peritonitis zu verschulden? Erstens erzeugt jedes Aetzmittel eine abakterielle Entzündung, die allerdings auf den Herd beschränkt bleiben muss, wenn nicht nachträglich Bakterien hinzukommen. Daraus folgt der Schluss, dass in allen Unglücksfällen Luftkeime oder andere Infectionsträger, sei es von unten, sei es vom Darm aus, ibren Weg zum Schorfe fanden.

Neben der Verhütung von Gefahren eröffnen die festgewonnenen Thatsachen einen weiteren Ausblick zu einer neuen Behandlungsart. Nachdem wir wisson, dass selbst in die am Fransenende verschlossenen Eileiter wässerige Flüssigkeiten einzudringen vermögen, so bald nur ihr Ostium uterinum durchgängig ist, vermögen wir selbst eine Behandlung von Erkrankungen der Tube methodisch einzuführen, wo wir dies wollen. Es ist selbstverständlich, dass hier als erstes Zicl die Bekämpfung der ascendirenden Gonorrhoe vorschwebt und es ist selbst hier im Anfang der Erkrankung, wenn die Verschwellung der Schleimhaut den Eileiter noch nicht undurchgängig gemacht oder später, wenn die Epithelien wieder regenerirt sind, mit wässerigen, nicht ätzenden, z. B. mit 2 proc. Argentaminlösungen möglich, die Krankheit zu bekämpfen und zu heilen. Wir haben damit bereits Jahre lang Versuche gemacht und sehr gute Erfolge erzielt. Wir möchten aber auch hierbei empfehlen, grosse Vorsicht zu üben und Anfangs nur mit kleinen Dosen 0,1-0,2 ecm der Lösung zu beginnen, weil der Uterus bezw. die Tuben individuell ausserordentlich verschieden reagiren und oft auf harmlose Eingriffe oder Arzueimittel Koliken mit Ohnmachtsanwandlungen eintreten können. Wir haben solche in der Klinik bei $1-2,5 \mathrm{ccm}$ Argentamin nie gesehen, ich persönlich jedoch auf $1 / 2 \mathrm{ccm}$ zweimal in der Privatsprechstunde und ist 
deswegen, bis man den Grad der Empfindlichkeit kennt, eine ambulatorische Behandlung zu widerrathen.

Diese Behandlung mit Argentamin wurde in der biesigen Klinik in der letzten Zeit in grosser Zahl durchgeführt und entweder täglich oder jeden 2. Tag 1-2,5 ccm mit der oben (S. 305 ) abgebildeten Spritze in den Uterus eingebracht. Danach traten regelmässig in der erkrankten Seite mässig starke Schmerzen auf, die 1-2 Stunden anhielten, niemals aber Koliken. In allen Fällen mit Ausnahme eines einzigen, der sich nach wenig Tagen der weiteren Behandlung entzog, trat schnelle Besserung ein; in der Mehrzahl der Fälle konnten die Kranken nach durchschnittlich 3 wöchentlicher Behandlung als geheilt entlassen werden. Mehrere fühlten sich schon früher subjectiv so wohl, dass sie um die Entlassung baten. Einige blieben ungebessert und wurden operirt.

In dieser Hinsicht kann ich verweisen auf Versuche und Ergebnisse zweier russischer Autoren Grammatikati ${ }^{1}$ ) und G. J. Lebedeff $^{2}$ ), welche eine ähnliche Behandlung zu dem gleichen Zwecke sehon vor mir ausgeführt hatten. Wenn auch damals der Beweis noch fehlte, so schwebte doch schon wegen der Erscheinungen in einzelnen Fällen die Vermuthung vor, dass die eingespritzte Flüssigkeit durch die Tuben bis in die Bauchhöhle fliessen könne.

Grammatikati and Lebedeff benützten za ihren Injectionen Tinctura Jodi und Spiritus vini (97pCt.) aa 25,0 und Alumnol 2,5, machten die Injectionen täglich, doch in der Regel nicht ambulatorisch. Der Letztere giebt eine genauere Beschreibung. Er führte in den ersten Einspritzungen die Mündung der Kanäle wenig über den inneren Muttermund ein und drückte zuerst nur einige Tropfen aus der Spritze. Dann wurde von Tag zu Tag die Menge der Flüssigkeit vermehrt, immer streng darauf geachtet, dass der innere Muttermund weit sei, um einem Abfluss des Ueberschusses kein Hinderniss zu bereiten und in allen Fällen, wo der Halskanal eng war, mit Hegar'schen Dilatatorien bis zu

1) Intrauterine Injectionen als Behandlungsmethode der Entzündung der Geb.. d. Adnexe u. d. Beckenperit. Ref. Centralbl. f. Geb. 1896. S. 1229.

2) Ueber die Bebandlung der Entzündungen des Uterus und seiner Adnexe durch intrauterine Injectionen. Centralbl. f. Geb. 1899. S. 833 u. ff. Hier ist auch die ältere Literatur citirt; vergl. auch Walton, Contributions à la étude de la pelvi-péritonite. Académie Royale de médecine de Belgique. 1887. Juli. 
$\mathrm{Z}_{\mathrm{w}}$ eifel, Die Gefahren und der Nutzen der intrauterinen Injectionen. 313

No. 6 und 7 erweitert. Die grösste Dosis war $2 \mathrm{ccm}$. Nach der Einspritzung mussten die Kranken noch $1 \frac{1}{2}$ Stunden ruhig sitzen oder liegen bleiben.

Von grossem Interesse würde es sein, Kenntniss zu erhalten über die Verhältnisse beim puerperalen Uterus, weil hierbei der Wansch einer unmittelbaren Einwirkung bei Entzündungen oft nahe liegt.

Ein einziger Versuch, den ich machen konnte, fiel negativ aus, sodass vorläufig die Frage beim frisch puerperalen Uterus noch offen ist. 